\title{
Médiévales
}

Langues, Textes, Histoire

73 | automne 2017

Le texte à l'épreuve du numérique

\section{Pour des éditions numériques critiques. L'exemple des textes français}

For Critical Scholarly Digital Editions. The Example of Medieval French Texts

\section{Frédéric Duval}

\section{(2) OpenEdition \\ 12 Journals}

\section{Édition électronique}

URL : https://journals.openedition.org/medievales/8165

DOI : 10.4000/medievales.8165

ISSN : $1777-5892$

Éditeur

Presses universitaires de Vincennes

\section{Édition imprimée}

Date de publication : 15 décembre 2017

Pagination : 13-29

ISBN : 978-2-84292-801-8

ISSN : 0751-2708

\section{Référence électronique}

Frédéric Duval, «Pour des éditions numériques critiques. L'exemple des textes français », Médiévales [En ligne], 73 | automne 2017, mis en ligne le 28 février 2019, consulté le 22 avril 2022. URL : http:// journals.openedition.org/medievales/8165; DOI : https://doi.org/10.4000/medievales.8165 
Frédéric Duval

\section{Pour des éditions numériques critiques. L'exemple des textes français}

Depuis l'Éloge de la variante de Bernard Cerquiglini ${ }^{1}$, qui voyait - après Roger Laufer - l'avenir de l'édition dans le multi-fenêtrage, et depuis le début contemporain du Princeton Charrette Project dirigé par Karl D. Uitti ${ }^{2}$, presque trente ans ont passé. Si les textes en français médiéval disponibles en ligne se sont multipliés, force est de constater que les éditions numériques natives demeurent particulièrement rares, surtout au sens que leur donne Peter Robinson ${ }^{3}$. Le recours au numérique, il est vrai, s'impose moins pour les textes médiévaux que pour des dossiers génétiques ${ }^{4}$, et la communauté des médiévistes se contente d'éditions conçues pour le papier, parfois numérisées. Du côté des textes littéraires écrits en français, le bilan éditorial est bien maigre : on peut citer des publications numériques proches de l'édition papier, généralement réalisées par un seul chercheur avec l'aide d'une cellule de publication numérique, comme à l'École des chartes ${ }^{5}$ ou aux Presses universitaires de Caen ${ }^{6}$. Les potentialités du numérique y sont plus ou moins développées. Du côté des projets d'équipe, plus ambitieux,

1. B. CERQUIGLINI, Éloge de la variante. Histoire critique de la philologie, Paris, 1989.

2. <https://www.princeton.edu/ lancelot/ss/>.

3. P. RobInson, «What is a Critical Digital Edition ? », Variants. The Journal of the European Society for Textual Scholarship, 1 (2002), p. 43-62.

4. Les dossiers génétiques rassemblent l'ensemble des témoins écrits documentant le processus d'écriture d'un texte. Ils sont exceptionnels au Moyen Âge, où les brouillons n'étaient pas conservés. Il faut attendre la seconde moitié du XVIII ${ }^{\mathrm{e}}$ siècle pour que ce type de dossier se multiplie.

5. <http://elec.enc.sorbonne.fr/>, par exemple Le Livre de jostice et de plet, éd. G. PASTORE, <http://elec.enc.sorbonne.fr/josticeetplet/>.

6. Guillaume de SAINT-PAIR, Le Roman du Mont Saint-Michel, éd. C. BougY, $<$ https://www.unicaen.fr/services/puc/sources/gsp/>. 
outre le projet Charrette, on peut citer le Online Froissart $(2007)^{7}$, le Partonopeus de Blois ${ }^{8}$, la Queste del saint Graal $^{9}$, ainsi que la Roman de la Rose Digital Library ${ }^{10}$, le Mystère des actes des apôtres ${ }^{11}$ et le projet Aspremont ${ }^{12}$. La plupart ne visent pas à donner d'éditions critiques : le projet Charrette se présente ouvertement comme une «archive "; Partonopeus de Blois, Roman de la Rose et Online Froissart excluent d'emblée cet élément. Quant à la Queste, elle envisage l'édition critique une fois transcrits tous les manuscrits, alors qu'à ce jour un seul a été traité.

S'il est hautement souhaitable que les éditions numériques de textes français médiévaux se développent, il faudrait éviter qu'elles suivent aveuglément la pente du digital scholarly editing anglo-saxon ${ }^{13}$. L'essor de l'édition numérique passe, certes, par une prise en compte des potentialités du nouveau médium et donc par un éloignement du modèle papier, mais la mise à distance du modèle traditionnel doit s'accompagner d'un regard critique porté sur les pratiques numériques dominantes, dont les soubassements théoriques ne s'accordent pas toujours avec la réalité textuelle médiévale. Cette contribution, trop rapide, voudrait sensibiliser les médiévistes francisants à l'intensité actuelle des débats théoriques consacrés à l'édition numérique et à la place que peut y trouver l'édition critique.

\section{Entre définitions, glissements de sens et malentendus}

Ces dernières années ont vu plusieurs tentatives de définition de l'édition savante numérique. La tradition anglo-saxonne domine et les concepts, exprimés en anglais, se parent de nuances difficilement perceptibles par les non-anglophones, comme la distinction entre digital scholarly edition et scholarly digital edition. En outre, cette terminologie naissante est déconnectée des traditions ecdotiques française ou néo-lachmannienne, largement ignorées, ce qui rend les débats parfois compliqués à suivre.

7. The Online Froissart, version 1.4, éd. P. AINSWORTH et G. CROENEN, Sheffield, $<$ https://www.hrionline.ac.uk/onlinefroissart/>.

8. Partonopeus de Blois, Sheffield, <http://www.hrionline.ac.uk/partonopeus>.

9. Queste del saint Graal, éd. C. MARCHELlO-NIZIA et A. LAVRENTIEV, Lyon, Équipe BFM. Publié en ligne par la Base de Français Médiéval, <http://portal.textometrie.org/ $\mathrm{bfm} /$ ?command=documentation \&path=/GRAAL $>$.

10. <http://romandelarose.org/>.

11. Simon GréBAn, Le Mystère des Actes des Apôtres, CNRS/Lamop (UMR 8589), $<$ http://eserve.org.uk/anr/>.

12. <http://www.chansondaspremont.eu/index.html>.

13. Saluons à cet égard le récent D. APOLlon, C. BÉlise et P. RÉGNIER éd., Digital Critical Editions, Urbana, 2014, qui fait entendre en anglais la voix différente de dixneuviémistes français et norvégiens. La récente thèse de J. CASENAVE, La Transposition numérique de l'édition critique : éléments pour une édition de l'Isopet 1-Avionnet (Université Paris-est Créteil et Université de Montréal, soutenue le 28 octobre 2016) replace également la discussion dans une tradition philologique romaniste. 
La place de la « critique » s'est affirmée comme un enjeu important des digital scholarly editions afin d'asseoir leur statut académique à côté des éditions critiques traditionnelles où l'adjectif « critique » implique une prise en compte de la tradition textuelle, qu'il s'agisse d'une édition reconstructionniste ou d'une édition conservatrice. L'habile définition donnée par Patrick Sahle («Une édition scientifique est la représentation critique d'un document ou d'un texte historique $\left.{ }^{14} »\right)$ prend bien soin d'inclure la dimension critique, dans une conception large et ouverte : «Pour le dire brièvement, on peut considérer que le terme "critique" contient toutes les activités qui appliquent le savoir scientifique et le raisonnement au processus de reproduction des documents et de transformation d'un document ou d'un texte en une édition ${ }^{15}$. L'encodage d'une transcription est partie prenante du processus critique, tout comme la modélisation du projet éditorial. Dans ces conditions, la critique textuelle, au sens de la Textkritik, peut être considérée comme subsidiaire au sein de l'activité critique de l'éditeur.

Le discours anglo-saxon consacré au digital scholarly editing, à l'instar de la définition de Patrick Sahle, s'appuie sur la triade document, text, work. Si les avis des théoriciens de l'édition numérique divergent sur l'orientation de l'édition vers l'une ou l'autre de ces entités, les dénominations comme la tripartition font désormais consensus ${ }^{16}$. De fait, les éditions numériques ont été contraintes d'articuler plus nettement que les éditions papier ces trois niveaux pour structurer des données toujours plus abondantes. L'attention renouvelée au document était soutenue à la fois par des positions théoriques (comme celle de la material philology) et par de nouvelles pratiques (comme l'association de reproductions photographiques à la transcription de témoins). En outre, l'encodage conduit à distinguer ce qui relève de la matérialité (i.e. du document) de ce qui tient au contenu et à la structure intellectuelle (i.e. au text et au work). Quant à l'œuvre, elle peut être représentée par plusieurs textes. Là encore la distinction est tout à la fois théorique (voir par exemple les conceptions du social text) et pratique : en génétique textuelle, des textes peuvent précéder l'œuvre. La scholarly digital edition, telle qu'elle est généralement définie, ne concorde donc pas avec l' « édition de texte(s)», mais tend à se présenter

14. «A scholarly edition is the critical representation of historical documents and texts » (P. SAHLE, «What is a Scholarly Digital Edition? », dans M. J. DRISCOLL et E. PIERAZZO éd., Digital Scholarly Editing : Theories and Practices, Cambridge, 2016,p. 19-39 (p. 23), <http:// dx.doi.org/10.11647/OBP.0095.02>). L'auteur synthétise dans cet article des développements publiés dans P. SAHLE, Digitale Editionsformen : zum Umgang mit der Überlieferung unter den Bedingungen des Medienwandels, Norderstedt, 2013.

15. «To sum up, we may take the word critical as a container for all those activities that apply scholarly knowledge and reasoning to the process of reproducing documents and transforming a document or text into an edition » (Ibid., p. 24).

16. Pour un panorama récent des différentes positions, $c f$. B. BORDALEJO éd., «Work and document », Ecdotica : rivista di studi testuali, 10 (2013). 
comme édition de document(s), une documentary scholarly digital edition. On assiste donc à des glissements sémantiques : work désigne le texte de l'auteur, éventuellement le texte correspondant à la volonté de l'auteur, et implique la notion d'authenticité ; text dénomme la séquence linguistique attestée dans un document transmettant l'œuvre ; enfin document est une manifestation physique d'un text. Alors que la critique textuelle se concentre sur les relations entre texte (work) et témoin (text), la scholarly digital edition tend à privilégier les relations entre text et document. Le décalage, on le voit, est à la fois conceptuel et terminologique.

\section{Le triomphe des éditions orientées vers le document}

Rares sont donc les projets numériques d'édition de textes français médiévaux à proposer une édition critique. Certains ne se revendiquent d'ailleurs pas comme éditions mais comme archive ${ }^{17}$ ou library. Toutefois, la frontière entre archive et edition se révèle poreuse et labile : l'ampleur de la documentation accessible sur des sites consacrés à un texte (work) ou à un auteur peut justifier l'appellation archive, même pour des projets incluant une édition critique, à l'instar du projet Charrette. Indépendamment des questions terminologiques, les éditions numériques orientées vers le document ont pour l'instant triomphé.

La raison principale de leur succès, mainte fois commentée, est la coïncidence des nouvelles possibilités techniques offertes par le numérique avec des théories du texte à la mode, contestant le bien-fondé des éditions idéalistes et reconstructionnistes ${ }^{18}$. La critique acerbe des éditions éclectiques pratiquées dans la lignée de Greg et Bowers a trouvé une application positive dans la confection d'éditions ou d'archives numériques. La révolution du social text, dont Jerome McGann est l'un des principaux protagonistes ${ }^{19}$, conduisait à ne pas privilégier un état textuel (text) plutôt qu'un autre. Dans un grand élan de relativisme, l'orientation vers l'auteur et la notion même d'autorité se trouvaient contestées. La variance de Bernard Cerquiglini, qui nie la relation hiérarchique entre œuvre et texte, allait même

17. E. Vanhoutte, « Defining Electronic Editions : a Historical and Functional Perspective », dans Text and Genre in Reconstruction : Effects of Digitalization on Ideas, Behaviours, Products and Institutions, Cambridge, 2010, p. 119-144 (§ 32), <http://books. openedition.org/obp/654>.

18. Pour un exposé plus développé des raisons du succès des documentary editions, cf. E. PIERAZZO, «Digital Documentary Editions and the Others », Scholarly Editing, 35 (2014), <http://www.scholarlyediting.org/2014/essays/essay.pierazzo.html>.

19. Cf. J. J. McGann, A Critique of Modern Textual Criticism, Chicago/Londres, 1983. Depuis, J. J. McGann a pratiqué l'édition numérique et écrit nombre d'articles méthodologiques et théoriques à son sujet. $C f$., entre autres, «Electronic Archives and Critical Editing », Literature Compass, 7 (2010), p. 37-42, et « Philology in a New Key », Critical Inquiry, 39 (2013), p. 327-346. 
jusqu'à dissoudre l'œuvre dans un ensemble de textes d'identique valeur. Ces approches ont eu le mérite de pousser les chercheurs à s'intéresser de plus près aux documents. Parallèlement, l'inadéquation de l'édition papier avec ce nouvel environnement théorique s'est affirmée : pour des raisons économiques, le papier est contraint de se limiter à un seul état textuel (single text editions, one text editions), qu'il soit ou non reconstruit. Grâce au «multi-fenêtrage » et à l'hypertexte, le numérique s'est imposé comme le médium approprié et indispensable à l'application des « nouvelles » théories textuelles.

Du point de vue théorique, deux éléments principaux ont contribué à positionner le curseur du côté du témoin (document) : d'une part, le relativisme qui rend caduque la notion d'autorité dont jouirait une version du texte par rapport aux autres ; d'autre part, le discrédit des éditions reconstructionnistes (ou éclectiques), au motif qu'elles donnent à lire un texte qui n'a jamais existé. Les données sont désormais préférées aux hypothèses ${ }^{20}$. En plaçant les data au centre du dispositif d'archive et/ ou d'édition, ces théories se rattachent nettement à la longue tradition des méthodes ecdotiques cherchant à bannir l'interprétation de l'édition. Le texte édité est « objectivisé », libéré des conjectures liées à une intention de l'auteur considérée comme inatteignable. L'essentiel est de rendre ce qui se trouve sur la page ${ }^{21}$. Il est certain que la « dictature » de l'authenticité auctoriale a bien souvent conduit à ignorer des faits culturels, linguistiques, d'histoire des textes, en privilégiant les seuls éléments utiles à la reconstruction. Grâce à des approches quantitatives et comparatives, des champs d'études s'ouvraient et les questionnaires scientifiques se renouvelaient : la linguistique diachronique ou d'états anciens en a profité, notamment en syntaxe, par l'étude de la ponctuation ou de la segmentation graphique. L'étude des microvariantes est un secteur en développement ${ }^{22}$ et l'encodage toujours plus poussé des documents, notamment allographétique, promet de nombreuses découvertes.

À côté des aspects théoriques et technologiques, le succès des éditions documentaires tient à la transparence et au caractère cumulatif du numérique. Par la force des choses, l'édition savante papier doit opérer une sélection au sein des informations recueillies au cours du travail. L'édition numérique permet de publier des données de départ (comme des

20. Sur la position de Contini et plus largement sur celle des néo-lachmanniens, $c f$. L. LEONARDI, « Il testo come ipotesi (critica del manoscritto-base) », Medioevo romanzo, 35 (2011), p. 5-34.

21. Voir à ce sujet les réserves de P. RoBInSON, « Towards a Theory of Digital Editions », Variants, 10 (2013), p. 105-131 (p. 113).

22. Plusieurs études de ce type sont présentées dans Y. G. LePaGE et C. MiLAT éd., Por s'onor croistre. Mélanges de langue et de littérature médiévales offerts à Pierre Kunstmann, Ottawa, 2008. 
reproductions photographiques), des données intermédiaires, notamment les transcriptions, partielles ou complètes de différents témoins, les résultats de collation, etc., mais aussi des données facilitant l'interprétation des textes ou des documents, comme des notices de manuscrits, des contributions scientifiques... François Masai a jadis affirmé que l'édition diplomatique était une étape indispensable à la confection de toute édition critique ${ }^{23}$. À ce titre, les éditions documentaires peuvent être considérées comme des étapes préliminaires à la réalisation d'éditions critiques, même si elles n'entrent pas dans le projet initial d'édition.

La confection d'éditions documentaires se révèle de facto moins complexe que celle d'éditions critiques reposant sur un socle d'éditions documentaires. L'effort de standardisation de l'encodage, notamment dans le consortium TEI, a beaucoup porté sur le balisage des témoins, alors que le balisage de textes hypothétiques et reconstruits, articulés avec différents témoins, n'a pas encore trouvé de solutions satisfaisantes. En outre, à partir d'un cahier des charges et d'une documentation bien rédigée, il est possible de partager le travail d'édition documentaire, alors que la pesée critique de chaque leçon au sein d'une tradition est moins aisément mutualisable. La production d'un texte critique est particulièrement chronophage et génère quantitativement peu de données par rapport aux éditions documentaires. Les systèmes d'évaluation et de financement de la recherche ont tendance à favoriser les éditions documentaires, qui permettent d'atteindre à moindres frais des masses critiques justifiant les dépenses.

L'édition numérique est encore au berceau. Si l'édition documentaire prédomine, les positions évoluent : Peter Robinson a ainsi admis qu'il s'était trompé en abandonnant « l'édition d'un seul texte dans son édition de The Wife of Bath's Prologue en faveur d'un ensemble de visions différentes du texte ${ }^{24} \gg$. La relation entre éditions orientées vers l'auteur (work) et éditions documentaires agite les éditeurs numériques, mais les philologues savent bien que cette tension est indépendante du passage du papier au numérique. L'affrontement en partie fantasmé entre Karl Lachmann et Joseph Bédier relève du même problème, que l'on voit déjà pointer à propos des textes homériques dans l'opposition entre Pergame et Alexandrie. Il est naturel que pour des raisons techniques (plus grande facilité), théoriques (théories textuelles en vogue) et scientifiques (nouveaux faits appréhendables via des comparaisons automatisées et des recherches quantitatives modifiant à leur tour le questionnaire des chercheurs), les éditions documentaires aient été jusqu'ici favorisées.

23. F. MASAI, « Principes et conventions de l'édition diplomatique », Scriptorium, 4 (1950), p. 177-193.

24. «[...] the single (edited) text in the edition of The Wife of Bath's Prologue in favour of a set of different views of the text » $(c f$. E. VANHOUTTE, « Defining Electronic Editions... », $\S 1)$. 
Sans doute est-il trop tôt pour penser, avec Peter Robinson, que l'écrasante prédominance des éditions documentaires constitue un danger. Il convient cependant de se montrer vigilant, car il ne faudrait pas qu'une pratique hégémonique conduise à présenter comme seules acceptables les théories textuelles compatibles avec cette pratique : «La domination du document comme modèle de l'édition textuelle dans le domaine numérique suggère qu'est en train d'apparaître une théorie de l'édition numérique, fondée sur la transcription fidèle de documents individuels, qui affirme que l'édition numérique devrait se concentrer sur les seuls documents ${ }^{25}$. »

\section{Ontologie des textes et critique textuelle}

G. Thomas Tanselle a rappelé avec raison que le changement de médium n'altérait en rien l'ontologie des textes. La question fondamentale demeure le choix entre l'orientation vers l'auteur (ou toute autre autorité) et l'orientation vers le document ${ }^{26}$. De manière secondaire s'y adjoint la question de savoir si le médium (papier ou numérique) doit ou non intervenir dans la théorisation du texte. Pour ma part, il est clair que, si le numérique doit modifier les méthodes d'édition, voire la conception de l'édition, il ne doit pas modifier la conception du texte édité, qui n'a rien à voir avec elle. À dire vrai, les éditeurs ont toujours eu tendance à sous-estimer le fait qu'ils devaient articuler deux textualités différentes, celle du texte édité et celle de leur édition. Le numérique implique évidemment une nouvelle textualité de l'édition, mais ne modifie pas celle du texte édité.

\section{Textes ou données ? Lecteurs ou utilisateurs?}

La textualité de l'édition numérique est problématique : a-t-on encore affaire à un texte ou bien plutôt à un hypertexte ? Même si l'hypertexte est en gestation dans les tables, index et diverses aides à la lecture de consultation déjà présentes dans le codex médiéval, l'hypertexte numérique conduit à un fonctionnement cognitif distinct de celui de la lecture suivie. Or les théories

25. «The dominance of the document model of textual editing in the digital realm suggests that a theory of digital editions is emerging, based on page-by-page transcription of individual documents, which asserts that a digital edition should concentrate on the documents alone » (P. Robinson, « Towards a Theory of Digital Editions »..., p. 126). P. ShiLlingsburG, «The Impact of Computers on the Art of Scholarly Editing », dans G. EGAN éd., Electronic Publishing : Politics and Pragmatics, Tempe, 2010, p. 17-29, émet la même inquiétude.

26. «We still have to consider whether it is meaningful to pursue authorially intended texts or whether the documentary texts that survive from the past (perhaps purged of the obvious errors that can be identified) are the only texts we should study » (G. T. TANSELLE, « Foreword to Electronic Textual Editing (2006) », dans ID., Portraits and reviews, Charlottesville, 2015, p. 416-421 (p. 418) [publié d'abord comme « Foreword » dans L. BERNARD, K. O'BRIEN O’KeEFFE et J. UnsworTH éd., Electronic Textual Editing, New York, 2006, p. 1-6]). 
dialogiques du texte, dominantes aujourd'hui, reposent sur une lecture idéalement continue : le texte sans lecteur n'existe pas ; le sens du texte est une construction du lecteur à partir d'une suite de présuppositions validées ou infirmées par la séquence linguistique.

L'édition numérique offre naturellement des textes à lire de façon suivie et souvent même des exports imprimables, tout comme un lecteur d'édition papier peut consulter simultanément deux éditions d'un même texte, des reproductions des manuscrits édités et recourir à un dictionnaire, pratiquant ainsi une lecture qui se rapprocherait de la consultation hypertextuelle. Toutefois, à l'heure actuelle, le médium papier se prête mieux à la lecture suivie et le médium numérique à la consultation ${ }^{27}:$ d'un côté un « lecteur » confronté à un texte, de l'autre «l'utilisateur » consultant des données. Le chercheur qui est à la fois lecteur et utilisateur expert gagnera à pratiquer les deux média.

Grâce au numérique, de nombreuses données non interrogeables jusqu'à présent font l'objet d'enquêtes. Le questionnaire des chercheurs s'est affiné, tout comme le balisage, et des corpus homogènes se forment progressivement. Si l'édition documentaire a ouvert de nouveaux champs d'investigation en eux-mêmes, les données nouvellement accessibles ne modifient qu'à la marge le sens du texte édité. Leur intérêt est principalement de mettre à jour des structures cachées, des faits de système difficilement décelables à l'œil et à la main. Si les systèmes ainsi repérés font sens, ils influent peu sur le sens que l'on pouvait donner à un texte à partir d'une édition papier.

L'exploitation de nouvelles données explique en partie le succès des éditions documentaires, et ce à bon droit, mais il importe que les éditeurs numériques ne s'adressent pas qu'à des utilisateurs et que leurs éditions trouvent des lecteurs. Sinon, la césure entre lecteurs et éditeurs numériques risque d'être irrémédiable. À l'heure où l'ensemble du patrimoine textuel mondial est en cours de numérisation, il est de la responsabilité des éditeurs numériques de donner à lire des textes répondant à des critères académiques. Il y va de leur légitimité sociale et de la place des études textuelles dans leur ensemble.

Certains tenants de l'édition numérique documentaire dressent un constat paradoxal de l'édition critique traditionnelle, lui reprochant à la fois de présenter un clean text et d'être très difficile d'accès, surtout à cause de

27. $C f$. H. GABLER, « Theorizing the Digital Scholarly Edition », Literature Compass, 7 (2010), p. 43-56: «We read texts in their native print medium, that is, in books ; but we study texts and works in editions - in editions that live in the digital medium » (p. 46). Pour une mise au point sur la question, voir aussi K. S. G. RASMUSSEN, « Reading or Using a Digital Edition? Reader Roles in Scholarly Editions », dans Digital Scholarly Editing..., p. 119-133. 
l'apparat critique ${ }^{28}$. Il est certain que l'édition papier a véhiculé l'idé(ologi)e du « texte unique », alors que tout texte connaît des réalisations différentes au cours de son histoire. La synthèse (ou la réduction) opérée par l'édition critique en vue de présenter le texte au lecteur en simplifie d'ordinaire l'accès. La lisibilité des éditions électroniques n'a rien à envier à celle des éditions papier. Quand elles ne conservent pas un système d'apparat semblable à celui du papier, la liberté laissée au lecteur est problématique, car ce dernier ignore souvent les relations des textes qu'il compare ou leur place respective dans la tradition. Parfois, les interfaces ne sont pas intuitives et requièrent une longue familiarisation (On line Froissart) ; d'autres fois, des aides à la lecture systématiquement présentes dans les éditions papier disparaissent : c'est le cas de glossaires, remplacés au mieux par le renvoi au lemme d'un dictionnaire (par exemple On line Froissart ou le Mystère des Actes des Apôtres qui redirigent vers le Dictionnaire du moyen français), ou bien d'index. La simplicité ne doit en aucun cas être confondue avec un accès aisé à l'ensemble des matériaux.

L'accessibilité rejoint l'un des caractères saillants de l'édition numérique, son ouverture. Dans bien des projets, le lecteur est censé pouvoir se saisir des données documentaires pour construire son propre texte. En contrepoint, l'édition critique traditionnelle est fermée et la marge interprétative laissée au lecteur plutôt mince, puisqu'il ne dispose que d'un texte, accompagné d'un apparat critique sélectif impropre à restituer tous les témoins de la tradition ${ }^{29}$. Le lecteur-éditeur n'est pourtant qu'une chimère : le travail de recensio réclame une familiarité intense avec le texte et des connaissances philologiques pointues. En outre, rares sont à l'heure actuelle les éditions numériques médiévales ouvertes donnant la transcription de l'ensemble des témoins conservés, si bien que les données textuelles y sont tout aussi partielles que dans l'édition papier. La force du numérique est sa modularité : une édition numérique peut proposer des parcours plus ou moins ouverts, de la consultation libre d'un dépôt d'archives ${ }^{30}$ à des parcours commandées par des approches et intérêts divers (tradition du texte, approche générique, etc.).

28. Voir notamment E. PIERAZZO, « Modelling Digital Scholarly Editing : from Plato to Heraclitus », dans Digital Scholarly Editing..., p. 41-58.

29. La standardisation de la langue latine permet de viser l'exhaustivité. La récente édition numérique de GeOFFror Malaterra, Histoire du Grand Comte Roger et de son frère Robert Guiscard par M.-A. LUCAS-AVENEL a su en tirer profit dans une présentation novatrice de l'apparat, <https://www.unicaen.fr/puc/sources/malaterra/accueil>.

30. La liberté du lecteur était fondamentale dans les premières modélisations de l'édition électronique. Selon J.-L. Lebrave, « [l']innovation principale est peut-être que l'utilisateur devient partie prenante dans l'élaboration des matériaux qu'il consulte, et contrôle librement les cheminements qu'il effectuera à travers les documents » (J.-L. LEBRAVE, « L'écriture interrompue : quelques problèmes théoriques », dans N. CATACH éd., Les éditions critiques : problèmes techniques et éditoriaux, Paris, 1988, p. 126-142, ici p. 127). 


\section{L'altération des textes}

La philologie traditionnelle a toujours cherché à transcender les contingences matérielles et les corruptions engendrées par la transmission textuelle. Quelle que soit la textualité de l'édition, le texte édité a connu des copies qui l'ont éloigné de son état premier ou de l'état voulu par son auteur. Sans nier le processus d'altération-création de l'œuvre médiévale, il reste important de guider le lecteur afin qu'il sache ce qui s'éloigne certainement, probablement ou potentiellement de l'original et/ou de l'archétype. L'orientation vers l'auteur conserve son intérêt, d'autant que l'on a trop souvent souligné l'anachronisme des notions d'auteur et d'original, parfois contre les faits mêmes : le Moyen Âge a livré des éditions orientées vers l'auteur, a mis en place des systèmes de copie destinées à éviter l'altération des textes, qu'il s'agisse du système universitaire de la pecia ou du contrôle de la copie de ses œuvres assuré par Christine de Pizan. À cet égard, le relativisme posant des vérités globales du type « l'idée d'original n'existe pas $^{31}$ » ou « le texte n'existe pas $^{32}$ » est extrêmement dangereux, car les comportements médiévaux varient sensiblement d'un genre à l'autre, d'un auteur à l'autre, d'une langue à l'autre, d'un usage à l'autre, d'une époque à l'autre... Devant des altérations, il demeure légitime, y compris quand on postule plusieurs autorités, de chercher à remonter à un point précis de la tradition, et pourquoi pas à son point de départ ${ }^{33}$. Dans ce domaine, le potentiel du numérique reste à exploiter, car on peut concevoir plusieurs textes critiques, correspondant à plusieurs nœuds de la tradition.

La multiplication des éditions documentaires ne représente nullement un danger en soit pour le travail philologique. Au contraire, la transcription de l'ensemble des témoins permettrait dans l'idéal d'automatiser la collation et d'éviter une déperdition dommageable d'informations ${ }^{34}$. La menace tient plutôt à la rareté des éditions critiques numériques appuyées sur des éditions documentaires. Autrement dit, le lecteur n'est souvent pas en

31. F. LECOY, «L'édition critique des textes », dans A. VARVARO éd, XIV Congresso Internationale di Linguistica e Filologia Romanza, aprile 1974, 1978, t. I, p. 503 : « Je veux dire que le Moyen Âge n'a aucune idée de ce qu'a pu être un original et que c'est là un concept qui lui est totalement étranger, en ce sens qu'aucun lecteur ni aucun scribe n'a jamais eu le moindre scrupule - pour des raisons qui peuvent être variées - à retoucher ou à contaminer le texte qu'il lisait ou qu'il recopiait, ni, vraisemblablement, dans ses interventions, la moindre prétention à retrouver le texte primitif de l'œuvre qu'il pratiquait, s'il avait quelque doute concernant la leçon qu'il avait sous les yeux. »

32. B. Cerquiglini, Éloge de la variante..., en particulier p. 57-64. B. Cerquiglini excepte seule la Bible.

33. Sur ce point, $c f$. F. FISCHER, «All texts are equal, but... Textual Plurality and the Critical Text in Digital Scholarly Editions », Variants, 10 (2012), p. 77-92.

34. La fonction collate du Online Froissart est suggestive des possibilités de représentation de la variation textuelle. 
mesure de situer dans la tradition textuelle les leçons qui lui sont soumises. Cette lacune peut conduire à une exploitation erronée des documents, issus chacun d'un contexte de production spécifique impliquant des acteurs divers (atelier, copiste, commanditaire, libraire, etc.) et un modèle dont une partie des caractéristiques est héritée.

\section{Les textualités: histoire ou théorie?}

Que l'on considère avec D'Arco Silvio Avalle qu'il existe une vérité du texte et une vérité du document ${ }^{35}$, ou avec Cesare Segre qu'il s'agit de deux approches et non pas de «deux entités ontologiques ${ }^{36}$ », il faut admettre que les deux orientations éditoriales sont pertinentes. On a pu récuser l'édition critique au titre qu'elle ne correspondait à aucun témoin historique, à aucune version " usagée », et qu'elle n'était qu'une reconstruction artificielle. L'argument est surfait, car l'on compare deux régimes d'historicité différents. L'éditeur d'un texte médiéval ne peut se contenter de données brutes, sous peine de donner à lire un passé entièrement tributaire des hasards de la transmission et de la conservation. Le travail de l'historien consiste à reconstruire le passé : à cette fin il se doit d'échafauder des hypothèses. Pour connaître la vie de saint Louis, faut-il mieux lire Joinville ou la biographie de Jacques Le Goff ? Il en va de même pour les textes, la démarche philologique relevant pleinement de l'histoire.

Or l'histoire est sans doute la grande absente des débats qui animent le monde des éditions numériques. S'il a été bénéfique d'explorer des pans entiers de la culture textuelle délaissés à cause d'une orientation trop systématique vers l'auteur, la théorisation de l'édition numérique a surtout été élaborée par des chercheurs travaillant en philologie de l'imprimé ou en critique génétique (ou philologie d'auteur) ${ }^{37}$. Autrement dit, et en m'excusant de simplifier outrageusement, la théorisation s'est faite en considérant spécialement des époques où le texte de l'auteur est bien

35. D’A. S. AVALLE, « I canzonieri : definizione di genere e problemi di edizione », dans La critica del testo. Problemi di metodo ed esperienze di lavoro, Rome, 1985, p. 363-382 [rééd. dans D’A. S. AvAlle, La doppia verità : Fenomenologia, ecdotica e lingua letteraria del Medieovo romanzo, Florence, 2002, p. 155-173].

36. C. SEGRE, «Lachmann et Bédier. La guerre est finie », dans E. BUCHI, J.-P. CHAUVEAU et J.-M. PIERREL éd., Actes du XXVII congrès international de linguistique et de philologie romanes, Strasbourg, 2016, p. 15-28 (p. 25).

37. La méconnaissance des périodes antique et médiévale a conduit les théoriciens contemporanéistes à des affirmations parfaitement erronées. Ainsi, à titre d'exemple, Hans Gabler écrit que « The rationale of stemmatics came at a price. It made no allowance for the "fact of life" that variability is a natural condition of texts » (H. GABLER, « Beyond AuthorVentricity in Scholarly Editing », Journal of Early Modern Studies, 1 (2012), p. 15-35, ici p. 20). L'observation est fausse puisque c'est justement l'étude de la variation textuelle qui a abouti à l'élaboration de la méthode stemmatique. 
souvent une donnée. Les configurations antique et médiévale se distinguent des périodes plus récentes par la rareté des autographes et des copies autorisées par l'auteur, par l'écart chronologique entre la date de rédaction des textes et les plus anciens témoins conservés, mais aussi par un processus manuel de copie qui multiplie les risques de faute et d'altération du texte copié. Étant donné la place minoritaire des études antiques et médiévales dans le panorama littéraire et textuel global actuel, il ne faut pas s'étonner de la faible place réservée à la perspective reconstructionniste dans le discours théorique ${ }^{38}$. Tout projet d'édition numérique devrait prendre en compte les conditions de production du texte et le statut de l'auteur. La généralisation est ici impossible. Il convient d'éviter un double danger : celui du relativisme (tous les témoins n'ont pas la même valeur textuelle ; le texte fourni par un témoin donné ne peut être mis sur le même plan qu'un texte reconstruit); celui de l'idéalisme (le texte reconstruit par un éditeur en reste au stade de l'hypothèse et ne correspond jamais en tout point au texte de l'original ou à la «volonté de l'auteur »). S'il est problématique de « vouloir restaurer un texte sous une forme n'ayant jamais existé au Moyen Âge ${ }^{39}$ », il l'est tout autant de donner à lire, sous le nom d'un auteur, un texte éloigné de l'original. Que de commentaires littéraires attribuant à Chrétien de Troyes des éléments qui ne se trouvent que dans la copie de Guiot ! Comme ailleurs, le dogmatisme est à fuir : le texte à éditer est un produit humain, qui relève de l'histoire et n'est pas, en premier lieu, un objet théorique.

\section{Complémentarité des orientations éditoriales}

Le pluritextualisme favorisé par le numérique et prôné par un Cesare Segre tout comme par un Patrick Sahle amène à dépasser l'opposition entre approches documentaire et auctoriale. Il est désormais possible de les combiner au sein d'un même projet et de pratiquer l'une avant de l'enrichir par l'autre. Tout raisonnement théorique pour déterminer la meilleure approche est vain tant il faut tenir compte et des données textuelles et des paramètres économiques de l'édition.

En revanche, on n'ira pas jusqu'à affirmer que la dimension critique d'une édition documentaire est au moins égale à celle d'une édition orientée

38. Sur ce point, voir P. SAHLE, « What is a Scholarly Digital Edition ?»..., p. 39.

39. C. Marchello-Nizia, A. Lavrentiev, T. Rainsford, C. Guillot-Barbance, « La "philologie numérique" : tentative de définition d'un nouvel objet éditorial », dans Actes du XXVII congrès international de linguistique et de philologie romanes..., p. 1389-1400 (p. 1394). 
vers l'auteur ${ }^{40}$. Certes, la structuration des données et un balisage fin engagent une activité critique supérieure à celle d'une transcription papier ; en revanche, on ne peut nier que la prise en compte de la diachronie textuelle dans la pesée des variantes constitue un saut herméneutique supplémentaire. La configuration idéale est sans doute la complémentarité entre éditions documentaires et éditions critiques. Comme l'a bien montré Peter Robinson, cette complémentarité est d'ordre théorique autant que pratique :

On ne peut pas connaître l'œuvre sans les documents - de même, on ne peut comprendre les documents sans une compréhension de l'œuvre qu'ils génèrent. On peut en tirer le principe suivant : une édition scientifique doit éclairer autant que possible chaque aspect du texte, aussi bien le textecomme-œuvre que le texte-comme-document. Les éditions imprimées traditionnelles se sont davantage intéressées au premier. Un avantage évident des éditions numériques est qu'elles peuvent rééquilibrer le balancier en incluant un matériau bien plus riche pour l'étude du texte-comme-document qu'on ne peut y parvenir par le moyen de l'impression ${ }^{41}$.

Elle permet de combiner les deux approches du texte sans avoir à les articuler de manière hiérarchisée. Sans être nécessairement conçue comme un couronnement, l'édition critique peut donner du relief à la juxtaposition des éditions documentaires d'une même œuvre en servant, de manière optionnelle, de clef de lecture. Si le texte critique ne peut être que le fruit d'un travail de collation et de recension des textes documentaires, il constitue en retour un excellent point de départ pour accéder aux données documentaires. Il importe toutefois de distinguer l'élaboration scientifique, qui implique que l'édition documentaire précède l'édition critique, point d'arrivée, et la consultation optionnellement orientée du dossier textuel. Dans ce type de dispositif, l'édition critique n'est pas le produit de la culture du single text, mais une synthèse, une cartographie textuelle permettant de

40. C'est pourtant ce qu'affirment Mary-Jo Kline et Susan Holbrook Perdue, A Guide to Documentary Editing, Charlottesville, 2008, p. 3 : « Documentary editing, although noncritical in terms of classical textual scholarship, is hardly an uncritical endeavor. It demands as much intelligence, insight, and hard work as its critical counterpart, combined with a passionate determination to preserve for modern readers the nuances of evidence ». La citation est commentée dans E. PIERAZZO, Digital Scholarly Editing : theories, models and methods, Farnham/Burlington, 2015, p. 75 [2014, <hal-01182162>].

41. «One cannot know the work without the documents - equally, one cannot understand the documents without a comprehension of the work they instance. From this, a principle appears : a scholarly edition must, so far as it can, illuminate both aspects of the text, both text-as-work and text-as-document. Traditional print editions have focused more on the first. An evident advantage of digital editions is that they might redress this balance, by including much richer materials for the study of text-as-document than can be achieved in the print medium » (P. RoBINSON, « Towards a Theory... », p. 123). 
consulter les éditions documentaires tout en les situant dans la dynamique de la tradition. Pour reprendre la terminologie de l'édition génétique française, de telles éditions seraient verticales, car elles donneraient une vision en coupe de la tradition, mais elles n'interdiraient pas de considérer une synchronie textuelle à travers les éditions horizontales que sont les éditions documentaires.

Si l'édition critique peut à juste titre revendiquer une place au cœur du réseau hypertextuel de l'édition numérique, il convient de laisser au lecteur expert la possibilité d'entreprendre d'autres parcours, et en particulier la liberté de comparer des témoins sur d'autres critères que celui de la tradition textuelle ${ }^{42}$. Il est ainsi légitime de vouloir rapprocher des manuscrits de même provenance ou de même facture, illustrés dans le même atelier, etc. En outre, il ne faudrait pas que la synthèse orientée vers l'auteur en interdise d'autres : les versions vulgates ou les remaniements peuvent mériter également des synthèses critiques. Enfin, dans certaines traditions très éclatées, la synthèse critique sous forme d'édition critique traditionnelle est tout simplement exclue ${ }^{43}$.

Si l'édition critique n'a pas trouvé la place qui lui revient dans l'édition numérique, c'est que la complémentarité évoquée se heurte à de nombreux obstacles techniques, à commencer par la difficulté de tenir compte dans un même fichier XML à la fois du texte (work) et du document, en raison de l'impossibilité de chevauchement des séquences balisées.

Bien des réflexions restent à mener sur la modélisation et l'encodage des textes critiques. Marina Buzzoni a récemment pointé les insuffisances de la TEI pour l'encodage d'apparats dynamiques ${ }^{44}$. En l'état, les apparats demeurent souvent des dépôts de variantes, comme dans les versions papier, tout en étant très longs et complexes à baliser. Des outils sont à inventer pour améliorer ou remplacer un apparat critique dont l'encodage et la représentation sont trop souvent calqués sur le modèle du papier. Les potentialités du numérique sont encore largement sous-exploitées à

42. On peut toutefois s'interroger sur l'impact que peut avoir sur l'édition numérique de documents l'objectif de confectionner une édition critique. Il est vraisemblable que les choix d'encodage ne seront pas les mêmes que dans un projet uniquement documentaire. Cf. M. DAHLSTRÖM, « How Reproductive is a Scholarly Edition ? », Literary and Linguistic Computing, 19/1 (2004), p. 17-33 (p. 28).

43. $C f$. le projet d'édition numérique de la Chanson d'Aspremont.

44. "Encoding a long ("verbose") apparatus entry is most of the times extremely time consuming, very tricky and often frustrating, especially when information needs to be suppressed because of the lack of elements and/or attributes, or even because it is not always possible to break up the information into consistent base-units in order to keep all witnesses synchronised. In those cases, adding a note (in the $<\mathrm{rdg}>$ ?) would help - though not always ; yet, it seems like avoiding the problem rather than facing it » (M. BUZZONI, «A Protocol for Scholarly Digital Editions ? The Italian Point of View », dans Digital Scholarly Editing..., p. 59-82, ici p. 77). 
ce niveau : par des jeux de couleur, le texte critique pourrait devenir une véritable cartographie de la tradition ${ }^{45}$. Les corrections pourraient être affichées selon leur degré de probabilité ou selon le type d'argument qui les soutient (critique interne, critique externe).

Pour les textes en langue vernaculaire, la lemmatisation représente un enjeu central, car elle permettra d'automatiser efficacement la collation des témoins tout comme l'encodage des apparats, mais aussi d'aligner aisément témoins et édition critique. Alors que les recommandations de la TEI pour le balisage des éditions documentaires sont aujourd'hui au point, il revient aux chercheurs attachés à la confection d'éditions critiques de faire des propositions afin de les adapter aux possibilités (mais aussi aux contraintes) du numérique.

Comme l'a bien souligné Jerome McGann ${ }^{46}$, les chercheurs doivent protéger l'héritage du codex et transmettre les textes aux générations futures dans les meilleures conditions possibles. Leur responsabilité dans la transition numérique est grande. Or, l'on sait combien la numérisation s'effectue souvent dans de mauvaises conditions, entraînant la disparition de l'apparat critique des éditions anciennes ou au moins l'impossibilité de l'interroger. Pourtant, la consultation des textes à partir de versions numériques ou numérisées s'impose comme une évidence aujourd'hui, et l'on hésite parfois à se déplacer jusqu'à une bibliothèque. Afin d'éviter une solution de continuité dans la transmission et l'interprétation des textes, le développement d'une culture philologique minimale est indispensable. Il convient plus que jamais de faire comprendre aux lecteurs et utilisateurs que les textes qu'ils lisent sont des produits complexes, fruits d'interactions sociales, culturelles, techniques et économiques. Cette éducation du lecteur et de l'utilisateur serait facilitée par des sites présentant à la fois des éditions critiques et documentaires.

Pour l'instant, la réalité se rappelle à nous et l'édition numérique est à l'heure des choix. Les années 1990 ont vu l'émergence de modèles passant d'une conception idéaliste du texte critique à une conception idéaliste de l'édition numérique : "Idéalement, une édition scientifique "vraiment numérique" devrait être une édition qui vise à couvrir tous les aspects textuels, ou du moins autant que possible, d'une œuvre particulière,

45. Cf. l'édition citée de GeOFFroi Malaterra, Histoire du Grand Comte Roger et de son frère Robert Guiscard. Gianluca Valenti (« Una proposta per la pubblicazione (e la lettura) intuitiva delle edizioni di testi a tradizione manoscritta », Cognitive Philology, 6 (2013), <http://ojs.uniroma1.it/index.php/cogphil/issue/view/754>) a également avancé des propositions qui restent à approfondir et à compléter.

46. J. MCGAnN, « Coda : Why Digital Textual Scholarship Matters ; or, Philology in a New Key », Critical Inquiry, 39 (2013), p. 274-288 (p. 276). 
et de la meilleure manière possible, en exploitant tout le potentiel du médium numérique, tout en conservant les exigences académiques les plus élevées ${ }^{47}$. » De telles éditions, qui comprendraient notamment une édition documentaire de l'ensemble des témoins associés à une édition critique, n'ont pas encore été confectionnées pour les textes médiévaux français, même si le projet Charrette s'en rapprochait.

Les contraintes économiques - qui s'imposent à l'édition papier et ont conduit à la production de textes critiques gommant en partie la mouvance des textes - régissent également l'édition numérique, dont les coûts de réalisation sont souvent bien plus élevés que ceux du papier lorsque l'on veut exploiter les potentialités du médium. L'édition documentaire numérique est chronophage si l'on souhaite pratiquer un balisage très fin : on le constate avec le projet novateur de la Queste del saint Graal, qui devait en théorie pouvoir déboucher, une fois tous les manuscrits transcrits, sur une édition critique. Conditionner l'édition critique à la transcription de l'ensemble des témoins est envisageable uniquement pour des textes courts à tradition peu fournie. Dans les autres cas, le choix d'éditions documentaires s'est généralement imposé. La décision était logique, appuyée à la fois par la théorie des textes et par le désir de fournir aux chercheurs des éléments inaccessibles dans les éditions papier disponibles. L'édition documentaire pouvait être conçue comme complément d'éditions papier déjà publiées. Faute d'éditions critiques appuyées sur des éditions documentaires, il devient pressant de concevoir des éditions critiques numériques exploitant le médium numérique tout en s'affranchissant d'une volonté d'exhaustivité dans le traitement des témoins.

Aujourd'hui, l'impératif de productivité scientifique favorise encore l'édition papier, plus rapide et, qui plus est, pérenne. Trente ans après le début du projet Charrette, il serait souhaitable que les textes médiévaux prennent le tournant du numérique, que l'accès à l'écran soit ou non associé à une publication au format papier. Pour ce faire, souhaitons que les éditeurs disposent rapidement d'outils leur permettant d'assurer le futur numérique de l'édition critique.

Frédéric Duval - École des chartes (Centre Jean Mabillon), Labex Hastec, PSL Research University (Paris Sciences \& Lettres)

47. «Ideally, a scholarly edition that is "truly digital" should be an edition that aims at covering all or, at least, as many of the textual aspects as possible of a particular work in the best possible way, by exploiting the full potential of the digital medium while maintaining the highest possible academic standards » (F. FISCHER, «All texts are equal, but... », p. 81). 


\section{Pour des éditions numériques critiques. L'exemple des textes français}

Trente ans après les débuts de l'édition numérique de textes médiévaux en français, le bilan est maigre et les méthodes éditoriales privilégient les éditions documentaires, dans la tradition anglo-saxonne du scholarly documentary editing. Cette contribution voudrait sensibiliser les médiévistes francisants à l'intensité actuelle des débats théoriques consacrés à l'édition numérique et à la place que peut y trouver l'édition critique. Après une mise au point terminologique, elle revient sur les raisons qui expliquent le triomphe des éditions numériques orientées vers le document, avant de rappeler que le numérique ne modifie pas l'ontologie des textes et ne disqualifie pas l'approche de la critique textuelle. Au contraire, une complémentarité est à trouver entre éditions documentaires et éditions auctoriales.

Édition numérique - humanités numériques - littérature française - texte (édition).

\section{For Critical Scholarly Digital Editions. The Example of Medieval French Texts}

Thirty years after the first digital edition projects for texts in Old and Middle French, the results are meager and editorial methods favor documentary editions, in the Anglo-Saxon tradition of scholarly documentary editing. This contribution aims to sensitize the medievalists interested in French texts and documentation to the current intensity of the theoretical debates devoted to the digital edition and the place that the critical edition can find there. After a terminological refinement, it returns to the reasons for the triumph of digital editions oriented towards the document, before recalling that the digital does not modify the ontology of texts and does not disqualify the approach of textual criticism. On the contrary, a complementarity is to be found between documentary editions and critical editions.

Digital Edition - Digital Humanities - French Literature - Text (Edition). 
